\title{
CLAUDIO ESTEVA Y LA INSTITUCIONALIZACIÓN DE LA ANTROPOLOGÍA EN EL ESTADO ESPAÑOL
}

ISIDORO MORENO

Universidad de Sevilla

ismoreno@us.es

Indudablemente, nadie podría discutirle a Claudio Esteva su papel central en la institucionalización de la antropología en el Estado español. La disciplina, o el interés por lo que sería una nueva disciplina, apareció en España en la segunda mitad del siglo xix y uno de sus reflejos fue la constitución en Madrid, Sevilla y algunos otros lugares de sociedades antropológicas no mucho después de las creadas en Londres o Nueva York. En la Institución Libre de Enseñanza también se proyectó su presencia, si bien apenas se desarrolló, y algunas corrientes de lo que en aquellos tiempos se conocía como folk-lore bebieron de las mismas fuentes que la antropología que estaba tomando forma en los países hegemónicos. No hay que olvidar que Antonio Machado Álvarez, el demófilo andaluz, impulsor de la sociedad El Folklore Andaluz y luego de la sociedad de corte confederal El Folklore Español, fue el traductor del primer manual con el título de Antropología, que había escrito Edward B. Tylor ${ }^{1}$.

1 Mis trabajos sobre este tema son numerosos. El inicial se publicó en el primer número de la revista Ethnica, fundada por Esteva, a la que luego habré de referirme (Moreno, 1971). 
Como es sabido, a pesar de la pronta aparición del interés por los saberes antropológicos - en algunos lugares sin conexión con las universidades, pero en otros, de la mano de profesores de historia natural o de derecho, al igual que ocurrió en los países de referencia-, la antropología no llegó a institucionalizarse como disciplina ni a formar parte de los planes de estudio universitarios hasta mucho tiempo después. Solo tuvieron alguna presencia la antropología física (o biológica) y una etnología entendida de forma reduccionista, como el acercamiento exclusivamente a los mal denominados pueblos primitivos y como disciplina auxiliar de la prehistoria. Incluso, en algunos planes de estudio de Historia, hubo una asignatura de Prehistoria y Etnología, siempre a cargo de un prehistoriador, las más de las veces sin formación antropológica alguna, en la que la etnología era una especie de apéndice para describir la vida social y costumbres de los que se conocían como nuestros contemporáneos primitivos, que representarían, supuestamente, una especie de pervivencias de la Edad de la Piedra. Yo mismo, durante tres o cuatro cursos de mi etapa como profesor no numerario, allá por los primeros años setenta del pasado siglo, impartí Prehistoria y Etnología en la Universidad de Sevilla como encargado de curso tras jubilarse el catedrático de Prehistoria e Historia Antigua que la había dictado durante mucho tiempo. Claro que hice lo propio invirtiendo el diseño de la materia, es decir, la convertí en una especie de introducción a la antropología general, que, todo hay que decirlo, resultaba más atrayente para los estudiantes de lo que había sido la asignatura hasta el momento.

No es este el sitio para profundizar en las razones por las que la antropología, se entienda al modo boasiano de las cuatro subdisciplinas -antropología física, arqueología, lingüística y etnología - o al británico y, en general, europeo - centrada en lo que en Reino Unido siempre se llamó antropología social y en Francia, hasta no hace mucho, etnología- carecía de un lugar en la Universidad española cuando ingresamos en ella como estudiantes las personas de mi generación. Desde luego, no fue ajeno a esta ausencia el régimen político franquista y su nacionalcatolicismo, hermético a cualquier planteamiento crítico sobre la sociedad y que consideraba a Darwin y a los evolucionistas como demonios no menos malditos que los encarnados en Marx o Bakunin. Pero el desierto 
que para la antropología era la Universidad se había instalado mucho antes que la dictadura nacida en el golpe de Estado militar-fascista de julio de 1936. La pérdida de las últimos colonias caribeñas y del océano Pacífico y la insignificancia y muy escaso interés económico que despertaban las colonias africanas del siglo xx explican, al menos en buena parte, la falta de apoyo oficial a unos conocimientos que se estaban aplicando y, por ello, impulsando, en las metrópolis colonialistas contemporáneas. Y como quiera que la diversidad etnonacional del Estado español no se reconocía (situación que, en gran medida, continúa) y, en cambio, se afianzó el nacionalismo de Estado español tras el denominado desastre del 98 - la España definida como nación única e indivisible que sigue siendo incluso doctrina constitucional hoy-, tampoco se consideró necesaria la antropología, por parte de los poderes del Estado, como medio para abordar dicha diversidad cultural e identitaria interna. En tiempos del franquismo parecía bastar la negación represora de la plurinacionalidad del Estado y la existencia de los Coros y Danzas de España de la Sección Femenina de la Falange mostraba una inocua diversidad folklórica como medios de tratamiento de esa diversidad. Al no reconocer la existencia de diversas culturas ni los problemas de integración política en el Estado de las etnonacionalidades diferentes a la castellana -Euskal Herria, Cataluña, Andalucía, Galicia, Canarias...-e ignorar las minorías étnicas (en especial, los gitanos), la antropología se contemplaba como un saber teórico sin aplicación práctica alguna, que, además, podía ser potencialmente peligrosa, política y moralmente, por su orientación evolucionista y relativista ${ }^{2}$.

Esta era la situación cuando Claudio Esteva decidió volver de México en 1956, una situación que se prolongaría aún varios años. Las razones concretas de su regreso no están demasiado claras (o eso me parece). Leyendo diversas declaraciones en entrevistas, sobre todo recientes, y en escritos de don Claudio, quizá podríamos tener la sensación de que se trató de una estrategia muy planificada con el definido objetivo de «fundar los supuestos de una antropología española que no fuera solo acadé-

2 Ante esta realidad, no tiene ningún sentido que antropólogos que conocen al menos relativamente bien la antropología en el Estado español como Stanley Brandes defiendan que en el franquismo esta floreció (Brandes, 2011; Moreno, 2012b). 
mica...», como él mismo declaró. Pienso que la realidad sería más compleja, aunque la idea sí podría haber sido, desde el primer momento, la de intentar promover la instauración de la antropología, especialmente en la Universidad, tal como sucedía en México y también, aunque con menos vigor, en otros países de América Latina.

Esteva tendría que esperar nueve años para ver realizada la primera etapa del proyecto, con la creación de la Escuela de Estudios Antropológicos: una institución no universitaria ligada al Museo Nacional de Etnología, en Madrid. Durante los años que se prolongan desde su vuelta a su nombramiento como director del museo —un museo prácticamente desconocido, sin visitantes ni presupuesto- se vio obligado a licenciarse y doctorarse de nuevo (lo que hizo en la Sección de Historia de América de la Facultad de Filosofía y Letras de la Complutense), ya que las dificultades burocráticas y las especiales relaciones entre España y México (que seguía reconociendo el Gobierno de la República en el exilio) complicaban la convalidación de sus titulaciones mexicanas. Para sobrevivir, logró que lo contratasen como investigador para realizar informes en Guinea Ecuatorial y en la comarca de Sayago, en Zamora, mientras colaboró sin remuneración en la Revista de Indias, del Consejo Superior de Investigaciones Científicas (CSIC). A pesar de su habilidad y proverbial mano izquierda, no lo tenía fácil dados sus antecedentes políticos, pues había sido miembro de las Juventudes Socialistas Unificadas de Cataluña, estuvo recluido varios meses en el campo de concentración de Saint Cyprien, en Francia, al final de la guerra y fue refugiado político en México durante más de quince años. Desde luego, no era un hombre que despertara confianza al régimen. Pero, con su facilidad dialéctica logró acercarse a algunas autoridades académico-políticas del momento, como Gratiniano Nieto o Manuel Ballesteros, a la vez que establecía contactos con profesores de universidad demócratas como José Alcina —formado como antropólogo en el Museo del Hombre de París y, desde 1959, catedrático en Sevilla de Historia de América Prehispánica y Arqueología Americana-, que sería su principal aliado, y Luis Pericot — catedrático de Prehistoria en Barcelona, que de alguna manera representaba cierta continuidad con la tradición de Bosch Gimpera. 
El Congreso Internacional de Americanistas de 1964 constituyó un importante paso en la visibilización académica de la antropología, que Esteva supo aprovechar posteriormente con habilidad. En España no había tenido lugar este congreso - en el que la antropología, en sus diversa ramas, seguía siendo el eje principal-desde los años treinta, en tiempos de la República, y su celebración en tierras españolas de nuevo encontró grandes dificultades, ya que se oponían muchos académicos de diversos países y buena parte de los exiliados españoles, que consideraban que podría interpretarse como un reconocimiento del régimen franquista, del que abominaban. Por otro lado, las autoridades franquistas, especialmente las académicas, también veían con gran desconfianza el desembarco de intelectuales, algunos de los cuales vivían en el exilio o incluso habían formado parte de las Brigadas Internacionales. El impulsor de aquel congreso y su secretario general, Alcina, logró convencer a los opositores del exterior, aunque apenas logró apoyo alguno oficial en el interior ${ }^{3}$. El congreso era itinerante, pues se inauguró en Barcelona, se desarrolló en su mayor parte en Madrid y las últimas sesiones, incluida la de clausura, se celebraron en Sevilla. No se trataba de un capricho, sino de la forma de hacer presente el americanismo en general; entonces en España esta disciplina - controlada por gentes del movimiento o del Opus Dei y de muy escasa relevancia profesional - se centraba en la historia colonial y en la glorificación del imperio. Además, las tres ciudades se consideraban las más favorables para que la antropología naciera (o renaciera, si se quiere) en el ámbito universitario ${ }^{4}$.

\footnotetext{
3 Alcina pidió colaboración a algunos de quienes entonces éramos sus alumnos en tareas relativas al evento durante los dos días del congreso en Sevilla. Recuerdo que fui con varios de mis compañeros a la vieja estación de plaza de Armas para recibir a los congresistas que venían desde Madrid en tren y acompañarlos a los hoteles.

4 En Sevilla, en concreto, Alcina había fundado el Seminario de Antropología Americana, poco más que una placa en una pared y un membrete en un papel, pero que fue la base para la incorporación en los estudios de la especialidad de historia de América de una asignatura de Antropología y Etnología de América, que se añadió a la de Prehispánica (ambas impartidas por él). Alcina también era el referente de investigaciones de campo en Andalucía, especialmente mediante el proyecto Estudio Etnológico de Andalucía Occidental, para el que consiguió una pequeña subvención y que hizo posible, en los años 1963-1964, la presencia de un pequeño grupo de recién licenciados y estudiantes que pudimos introducirnos en la antropología bajo la supervisión del maestro y continuar para conseguir su institucionalización cuando él se trasladó a Madrid en 1967 (Alcina, 1964, 1972, 1989).
} 
El congreso, más allá de alcanzar estos y otros objetivos, fue la ocasión para que Esteva entrara en contacto con un buen número de académicos internacionales y también con personas del americanismo oficial español que, de una manera u otra, tuvieron relación con él. Sus dotes de relaciones públicas le permitieron poner en valor (como se dice ahora) su condición de antropólogo formado internacionalmente y dar a conocer sus proyectos y disposición para ponerlos en práctica. La situación del madrileño Museo Nacional de Etnología le facilitó por primera vez desempeñar un puesto institucional. La dirección que entonces ejercía Julio Caro Baroja era solo nominal y el museo se encontraba en un estado lamentable: faltaban más de 1500 piezas del inventario, tenía cortada la luz por falta de pago, no se había estipulado un sueldo para el director (solo una pequeña gratificación)... Pero Esteva tuvo la suficiente claridad de visión y voluntad como para comprometerse a reflotarlo aceptando una dirección en la que nadie estaba interesado y, sobre todo, para constituir en él su proyecto de una escuela de antropología. Como él mismo ha contado, logró convencer a Ciriaco Pérez Bustamante, personaje cercano al régimen y director del Instituto Gonzalo Fernández de Oviedo, del Consejo Superior de Investigaciones Científicas, para que firmara sus proyectos de la escuela y del Centro Iberoamericano de Antropología. De esta manera, logró que el Instituto de Cultura Hispánica (que dependía del Ministerio de Asuntos Exteriores y no del de Educación, donde no hubiera sido posible que desarrollara el proyecto) patrocinara la escuela y asumiera el centro; el instituto firmaba formalmente los títulos a los egresados y subvencionaba en cada curso a un máximo de 10 becarios iberoamericanos y otros 10 españoles, todos ellos posgraduados.

En unas pequeñas instalaciones algo modernizadas del museo, que contrastaban con las lúgubres y casi desiertas zonas expositivas, se impartieron durante tres cursos (1965-1966, 1966-1967 y 1967-1968) las clases de las diversas asignaturas - un puzle pluridisciplinario sin demasiada coherencia, todo hay que decirlo, aunque quizá no habría sido posible de otra manera en aquel momento- que constituían el curso de la Escuela de Estudios Antropológicos, que, además, se complementaba con una práctica de campo de unos dos meses de duración en comunidades del 
Pirineo de Huesca y cuyos resultados debían dar lugar a un informe, una especie de tesina, obligatorio para obtener el título.

Por la escuela pasamos, en alguno de sus tres años de funcionamiento, buena parte de quienes hemos formado, según nos han denominado, la «segunda generación de antropólogos», los ahora séniores, que ya nos hemos convertido en eméritos o hemos entrado en la jubilación forzosa. Procedíamos todos de Madrid, Barcelona o Sevilla y la presencia de alumnos latinoamericanos era poco significativa. ¡Quién elegiría, en los años sesenta, venir desde ese continente a Europa para estudiar antropología en un país donde tal disciplina prácticamente no existía!

En realidad, la formación que adquirimos en la escuela, donde hicimos casi de todo - medimos cráneos, estudiamos matemáticas y demografía, nos empapamos de la cultura azteca, realizamos prácticas museísticas o nos acercamos a la teoría antropológica一, no garantizaba una formación sólida ni quizá agregaba demasiado al reducido bagaje con que contábamos anteriormente, en mi caso, a lo aprendido en los dos cursos de Alcina y en sus seminarios en Sevilla. Sin embargo, sí reafirmó, al menos en la mayoría de quienes allí estuvimos, nuestra decisión de intentar convertirnos en antropólogos o antropólogas y la voluntad de continuar formándonos de la manera que entonces era aquí posible, esto es, en grandísima medida, autodidacta o aprovechando las pocas oportunidades de salir al extranjero. También nos sirvió para vivir una experiencia que había de ser inolvidable: la de un verano en el valle de Chistau (Gistaín) -yo concretamente, junto con Josefina Roma, en Plan, el pueblo que mucho después habría de hacerse famoso por los tiones (solterones) y la caravana de mujeres para resolverles, potencialmente, la forzada soltería-, Benasque u otros valles del alto Pirineo, en pequeños pueblos en los que actuamos según las directrices (o no tanto) de Esteva, quien aparecía cuando menos lo esperábamos en visitas que llamábamos de inspección.

Pero más allá de lo que significara la escuela para la formación de quienes pasamos por ella, sentados en su aulita cinco o seis horas diarias, de lunes a viernes, un curso completo, su mera existencia significó algo cuya importancia no debe minimizarse: fue la primera institución que de forma sistematizada y reconocida oficialmente impartió un curso 
de antropología para posgraduados, con una serie de materias teóricas y prácticas, un trabajo final obligatorio y un diploma oficial a su término (aunque no fuera expedido por el Ministerio de Educación). Con ella, la antropología comenzó a existir, más allá de ciertas asignaturas en algunas universidades. Fue el primer paso en su institucionalización. Y Esteva fue el motor, el alma y el eje indiscutible de ello. Tan vinculada estaba su persona a esta primera experiencia que cuando en 1968 se convoca por primera vez en una universidad española, precisamente en la de Barcelona, una cátedra —en ese momento se denominaba agregaduría - de Etnología y él, ya con 50 años, la consigue, la escuela desaparece. La ocasión le suponía salir de la precariedad y no depender de quienes tomaban decisiones con respecto a las instituciones, personas pertenecientes, evidentemente, al régimen franquista, aunque con intereses diversos. La desaparición de la escuela tuvo lugar, entre otras cosas, porque la figura de don Claudio era entonces irreemplazable.

Sería injusto, a mi entender, considerar que con su marcha a Barcelona (otro regreso) y conseguida su estabilidad personal, Esteva se desentendió de la institucionalización de la antropología más allá de Cataluña. No seré yo quien señale su nivel de responsabilidad, total a mi entender, en atraer a la antropología a varios excelentes estudiantes que se habían formado en otras disciplinas. Sin duda, fue obra suya que, según el innovador Plan Maluquer puesto en marcha en aquella universidad, la Antropología Cultural - la cátedra de Esteva tomó esta denominación desde 1972 - fuera asignatura troncal en primero y, por tanto, obligatoria para los cientos de estudiantes de la Facultad de Letras. Esto permitió la formación de un buen número de grupos, hasta once, y favoreció la incorporación de profesorado, aunque con la precariedad de la categoría de profesor no numerario de entonces, compuesto por varios jóvenes profesores que continuarían su formación (en gran medida autodidáctica) posterior como docentes e investigadores. Profesores a los que Esteva dio acogida más allá de que compartieran o no su marco teórico-metodológico (que la mayoría no suscribían), lo que era muestra de un espíritu de tolerancia y una generosidad muy poco frecuentes. 
También durante sus primeros años en Barcelona logró que el CSIC creara, bajo su dirección, el Centro de Etnología Peninsular y, sobre todo, una revista que, a partir de 1971, se publicaría regularmente: Ethnica, la primera revista de antropología en el Estado español, a la que se invitó a colaborar, no solo a quienes trabajaban con él en Barcelona, sino también a otros que habíamos estado en la escuela madrileña, además de a colegas de otros países. El establecimiento de Antropología Cultural en la Universidad de Barcelona y la revista fueron claves no solo para la visibilización y despegue institucional de la antropología en Cataluña, sino en todo el Estado.

Durante años, Esteva fue el único catedrático de Antropología Cultural en la Universidad española. Como tal, fue uno de los ponentes en la Primera Reunión de Antropólogos Españoles celebrada en Sevilla en enero de 1973, de cuyo comité organizador fui secretario. Aquel primer encuentro universitario respondió a la visión norteamericana y mexicana de la antropología, es decir, la que integraba diversas ramas o disciplinas. Así, hubo ponencias sobre antropología física o biológica, sobre arqueología (una arqueología antropológica que en España no estaba representada más que por Alcina y algunos de los discípulos que había logrado conseguir desde su traslado a Madrid en 1967, tras la creación allí de una Subsección de Antropología Americana), sobre etnología y antropología social, sobre etnohistoria y sobre antropología aplicada, esta última a cargo de Esteva. En realidad, en esas jornadas, que merecerían el calificativo de fundadoras, participó - $y$ me incluyo - un pequeño grupo de personas pertenecientes a dos generaciones. Las más significativas, tanto en aquel momento como para el ulterior desarrollo e institucionalización de la antropología, fueron Claudio Esteva, José Alcina y Carmelo Lisón (de la generación sénior) y Jesús Contreras, Ignasi Terrades, Joan Frigolé, Joan F. Mira y yo mismo (de la generación júnior).

Una segunda reunión tendría lugar en 1974 en Segovia y fue el grupo de Barcelona, formado en torno a Esteva, el que organizó el Primer Congreso Español de Antropología en un año tan clave a todos los efec-

5 Tras casi cincuenta años de aquello, resulta interesante revisar lo que allí se debatió. Es posible gracias a que tres años más tarde se publicaron las actas, así como un directorio (Jiménez, 1975). 
tos como 1977. Fue en él cuando la disciplina se reorientó a la europea y se centró prácticamente en la antropología cultural y social, y abandonó, de hecho, la pretensión de incluir disciplinas que, en casi todos los casos, son independientes de aquella en la tradición académica de nuestro continente. Podría decirse, utilizando un símil futbolístico (pienso que lo aceptaría Esteva dado que durante su juventud fue, entre otras muchas cosas, futbolista y entrenador de fútbol), que él sufrió en aquel congreso una derrota a domicilio, ya que la gran mayoría de los participantes, cuyo número multiplicaba al de las dos reuniones precedentes, no aceptaron la visión integral, boasiana, que él había defendido siempre. Y, a pesar de este rechazo, continuó apoyando a sus colaboradores y antiguos estudiantes y siguió desempeñando uno de los papeles fundamentales en el desarrollo de la institucionalización de la antropología, que él siempre denominó cultural por razones tanto teóricas como estratégicas y para evitar el riesgo de que la disciplina acabara fagocitada por la sociología o se convirtiera en una rama de esta.

Por su condición de primer y, durante unos años, único catedrático de Antropología, Claudio Esteva participó en tribunales de oposiciones a plazas de esta disciplina. Hasta bastante tiempo después, no hubo posibilidad de que los cinco miembros de estos tribunales fueran catedráticos de Antropología. Incluso cuando, en mi caso, obtuve en 1986 la primera cátedra de Antropología (entonces con el apellido Social) que se creó en Andalucía, aún no había ese número. Mucho menos en octubre de 1975, cuando en Madrid tuvieron lugar los ejercicios para una agregaduría (como seguían denominándose entonces las cátedras) para la Autónoma de Barcelona, en los que él era el único miembro catedrático de Antropología 6 .

\footnotetext{
6 Aquella oposición, que tuvo lugar en los días terminales del dictador, en medio de una enorme incertidumbre política, no se me olvidará jamás porque el candidato que finalmente ganó la plaza, a la que también nos presentábamos Joan Frigolé y yo, realizó contra mí, públicamente, lo que era conocido como la trinca (que hacía muchos años que no tenía lugar, al decir de quienes llevan la cuenta de esas cosas). En este caso, consistió en una denuncia pública de varios folios en la que se me acusaba de estar interesado en obtener la cátedra (agregaduría) con el objetivo de introducir ideas revolucionarias y no científicas en la universidad, ya que yo pertenecía -y eso era cierto- a un partido ilegal de izquierda radical que formaba parte de la Junta Democrática de España, en cuya Comisión Central Permanente yo representaba a ese partido durante aquellos meses. Parece que aquella denuncia partía del exterior, aunque pienso que ello no eximía de responsabilidad a quien
} 
Durante varios años, a partir de la instauración en 1983 de la especialidad de Antropología Cultural en las Facultades de Geografía e Historia de Sevilla y Barcelona, esta última liderada por Esteva, y con la continuidad de la Subsección de Antropología Americana en la Complutense, en torno a Alcina y sus discípulos madrileños, estas tres universidades fueron las únicas en que existió el título de Antropología, aunque todavía no como licenciatura. Y habría que añadir el núcleo de la Facultad de Sociología de esta misma universidad, del que era cabeza indiscutible Carmelo Lisón. Además de estos cuatro núcleos (en tres universidades), iban tomando fuerza y ampliándose otros más: en las Autónomas de Madrid y Barcelona, en el País Vasco, en Canarias..., que también aprovecharon la oportunidad de la reorganización que supuso la creación de licenciaturas de solo segundo ciclo, ya avanzada la década de los noventa, para instaurar oficialmente el título de Antropología Social y Cultural como una de ellas. En realidad, la gran mayoría de los departamentos y áreas de Antropología propusimos una carrera de dos ciclos, pero el ministerio, en aquel momento en manos del PSOE, impuso el título reducido, de solo segundo ciclo, y una lista excesiva de titulaciones de primer ciclo que permitían poder acceder a él. En las reuniones con la secretaria de Estado, Elisa Pérez Vera, ya no se encontraban los séniores. De hecho, sin apenas darnos cuenta, algunos nos estábamos convirtiendo en tales. La primera generación, muy especialmente Esteva, nos había cedido ya el testigo. Pero esta es otra historia que también merecería ser tratada, pero no es este el lugar ni el momento.

A don Claudio lo jubilaron de forma forzosa en 1986, al cumplir los 65 años. Alguna inteligencia genial desde el ministerio consideró que los profesores de universidades debían jubilarse, como todo el mundo, a esa edad, por más que pudieran estar en su más plena madurez intelectual y dispuestos a continuar en la docencia y la investigación. Igual le ocurriría pocos años después a Alcina. Siempre me ha parecido una barbaridad

aceptó ser su cauce con el objetivo de favorecer sus intereses personales. El tribunal se componía del presidente, un catedrático de Metafísica, y, como vocales, un psicólogo, un sociólogo que ocupaba cargos políticos en el régimen, Esteva y Alcina. Tres que no eran antropólogos y solo dos que sí lo eran; de estos, solo uno con esa titulación. Y, paradojas de la vida, el candidato ganador se declararía pocos años más tarde ¡marxista! 
cómo la Universidad se ha permitido, y sigue permitiéndose, el lujo de derrochar cerebros (aunque aquella medida se rectificó parcialmente, pues se volvió a la situación anterior, la actual, de prolongación voluntaria hasta los 70 años más la posibilidad de dos o tres años de continuidad como eméritos). Esteva se rebeló contra la estupidez burocrática y en cuanto tuvo oportunidad, gracias a la invitación de sus colegas mexicanos, retornó a México, donde continuó su actividad académica, principalmente en El Colegio de Jalisco, cosa que hizo prácticamente hasta su reciente muerte; sin embargo, nunca perdió del todo su contacto con Barcelona y con quienes fueron sus discípulos. Su vitalidad le impedía aceptar ninguna jubilación. «Yo no me he acabado», insistía siempre en sus declaraciones.

La lucidez mental —y la vehemencia- acompañó a Esteva hasta el final de sus días, como lo reflejan sus escritos y entrevistas últimas. En la que le hizo en 2009 en Guadalajara (México) David Lagunas —que fue estudiante en Barcelona y es actualmente profesor titular en Sevilla-y en la publicada en Periferia en 2011 refleja sin ambages cuáles eran sus posiciones. No le importa comprometerse cuando señala que «el antropólogo posmoderno se encuentra dominado por el narcisismo y el culto neurótico a la propia personalidad»; cuando alerta de cómo «la reflexividad se está convirtiendo en la razón de ser de la Antropología de forma obsesionante»; o cuando afirma que «una debilidad actual es el exceso de categorías (...) en detrimento de la calidad descriptiva de la etnografía, siendo esta el fundamento de la buena antropología». Confieso que, sin haber estado nunca en el ámbito teórico-metodológico de Claudio Este$\mathrm{va}^{7}$, estoy de acuerdo, básicamente, con estas consideraciones. Y también -siempre que no generalicemos demasiado, porque sería injusto- coincido con su comentario de que encuentra a los antropólogos españoles actuales «cada vez más descafeinados [...] y narcisos».

7 Las bases teórico-metodológicas de mi quehacer antropológico, al menos desde finales de los años ochenta, son los paradigmas de la matriz cultural identitaria y de la glocalización (Moreno, 2010). GEISA, el amplio grupo de investigación creado en aquellos años y del que fui investigador principal hasta mi jubilación forzosa en 2017, se construyó sobre esos dos ejes (Moreno, 2010). Mis planteamientos sobre cómo y para qué la antropología está contenida en diversos textos aparece, entre otras fuentes, en Moreno, 2011 y $2012 a$. 
Así nos va en la disciplina, aquí y ahora: titulaciones de Antropología Social y Cultural en un gran número de universidades (quizá en demasiadas), cientos de profesores de los que no estoy seguro, en muchos casos, si son o no conscientes del marco teórico-metodológico en que se sitúan, banalidad de muchos temas y publicaciones, debilidad de los trabajos de campo, acercamiento peligroso al periodismo, el trabajo social o la sociometría; en definitiva, pérdida de los atributos fundamentales de la antropología, desorientación, caos teórico, confusión entre metodología y técnicas, juego literario en lugar de búsqueda de explicaciones o, al menos, de interpretaciones plausibles... La mayoría de los congresos y los contenidos de gran parte de los artículos en revistas reflejan esta indudable crisis a la que se refiere don Claudio. Y esto, a pesar de que tengamos hoy miles de estudiantes de Antropología en nuestras universidades (y también miles de graduados en paro o que se dedican a actividades muy alejadas de esta).

Esteva siempre creyó en el carácter aplicado de la antropología. En una antropología no narcisista, sino dirigida al análisis y resolución de problemas. Pongo un ejemplo que tiene que ver mucho con nuestra realidad presente. En 2009, Esteva se refería a «dos temas irresueltos, el problema del archipiélago mexicano y del archipiélago español: el mismo problema de integración de los grupos indígenas en la sociedad nacional mexicana lo tenían también los catalanes, los vascos, los gallegos, incluso los andaluces». Yo lo diría de otra manera y no llevaría tan lejos el símil, pero coincido en que ambas situaciones refieren a una situación de plurietnicidad y de multiculturalidad. ¿Y cuál sería la solución o el cauce de convivencia respecto de las consecuencias políticas de estas, es decir, de la existencia de diferentes hechos nacionales? Para Esteva no cabe duda: «[...] la solución para España es la Confederación de Naciones Ibéricas» con explícito reconocimiento como tales de Cataluña, País Vasco, Galicia, Andalucía y Portugal. Esto declaraba en 2009 y señalaba, además, que dicha idea la profesaba ya desde su exilio mexicano, compartida con los otros integrantes del grupo Presencia Peninsular, que allí crearon. Con este planteamiento, que para él es el resultado del análisis antropológico cultural y de la consideración de la antropología como fundamentalmente apli- 
cada, quizá hoy sería visto don Claudio por gran parte del establishment, no solo político, sino también académico, como un radical o un rebelde peligroso. Estoy seguro de que a él no le importaría.

\section{Bibliografía}

Alcina, J. 1964. Memoria acerca del trabajo de investigación sobre «Etnología de Andalucía Occidental». Sevilla: sin publicar.

AlcinA, J. 1972. «La antropología americanista en España: 1950-1970», Revista Española de Antropología Americana 7: 1-18. Madrid: Universidad Complutense de Madrid.

Alcina, J. 1989. «Etnología de Andalucía Occidental. Un proyecto de investigación veinte años después», Homenaje andaluz a Julián Pitt-Rivers. El Folklore Andaluz 3: 79-90. Sevilla: Fundación Machado.

BRANDES, S. 2011. "El nacimiento de la antropología social en España», Lugares, tiempos, memorias. La antropología ibérica en el siglo xxI. León: Universidad de León, 27-50.

CluA, M. 2013. «La antropología tiene que desvelar, desnaturalizar. Entrevista a Isidoro Moreno», Periferia. Revista de Recerca i Formació en Antropologia 18. Barcelona: Universidad Autónoma de Barcelona.

CLUA, M. et alii 2011. «Entrevista a Claudi Esteva Fabregat, antropólogo». Periferia. Revista de Recerca i Formació en Antropologia 14. Barcelona: Universidad Autónoma de Barcelona.

Comelles, J. M. / Prat, J. 1992. «El estado de las antropologías. Antropología, folklore y nacionalismos en el Estado español», Antropología 3: 35-61.

Esteva, C. 1969. «La Etnología española y sus problemas», Actas del I Congreso de Artes y Costumbres Populares. Zaragoza: Institución Fernando el Católico, 1-40.

Esteva, C. 2002. «Entrevista». AIBR. Revista de Antropología Iberoamericana 24.

JimÉNEZ, A. (ed.) 1975. Primera reunión de antropólogos españoles. Sevilla: Universidad de Sevilla.

Lagunas, D. 2012. «Claudio Esteva Fabregat, interpelado», Biblio 3W. Revista Bibliográfica de Geografía y Ciencias Sociales XVII(974). Barcelona, Universidad de Barcelona. 
Moreno, I. 1971. «La Antropología en Andalucía. Desarrollo histórico y estado actual de las investigaciones». Ethnica 1:107-144. Barcelona: CSIC. Moreno, I. 1973. «El estudio etnológico de España», Homenaje al profesor Carriazo. Sevilla: Publicaciones Universidad de Sevilla, 221-240.

Moreno, I. 1975. «La investigación antropológica en España», Primera reunión de antropólogos españoles. Sevilla: Publicaciones Universidad de Sevilla, 325-333.

Moreno, I. 1981. «Primer descubrimiento consciente de la identidad andaluza (1868-1890)», Historia de Andalucía. Madrid-Barcelona, CUPSA-Planeta, 233-251. [También en Moreno, I. (ed.) 2008. La identidad cultural de Andalucía. Aproximaciones, mixtificaciones, negacionismo y evidencias. Sevilla: Centro de Estudios Andaluces].

Moreno, I. 1984a. «La antropología cultural en Andalucía: estado actual y perspectivas de futuro», Antropología Cultural de Andalucía. Sevilla: Consejería de Cultura de la Junta de Andalucía, 93-107.

Moreno, I. 1984b. «La doble colonizació de l'antropología andalusa i perspectives de futur», Quaderns de l'Institut Català d'Antropologia 5: 69-84. Barcelona: ICA-Pol-len Edicions.

Moreno, I. 1986. «Fieldwork in Southern Europe and Scientific Colonization: The Case of Andalusia», 13th European Congress for Rural Sociology. Abstract. Braga: ,196-198.

Moreno, I. 2010. «Matriz cultural identitaria y glocalización: los paradigmas de GEISA (Grupo para el Estudio de las Identidades Socioculturales en Andalucía», Trabalho, políticas públicas e estratégias empresariais. Fortaleza: Universidade Federal de Ceará, 11-22.

Moreno, I. 2011. «Los papeles posibles de la antropología en tiempos de glocalización», Revista Andaluza de Antropología 1. Recuperado de $<$ www.revistaandaluzadeantropologia.org/>.

Moreno, I. 2012a. «Crisis de los pilares de la modernidad, globalización mercantilista y activación identitaria. Los papeles posibles de la antropología hoy», Fronteras del iluminismo. México D. F.: UAM-Juan Pablos Editor, 49-76. 
Moreno, I. 2012b. «Reinventando la historia (para falsificarla). Sobre las relaciones entre antropología y franquismo», Pels camins de l'etnografia: un homenatge a Joan Prat. Tarragona: Publicacions URV, 213-221.

PRAT, J. (coord.) 1985-1986. «Trenta anys de literatura antropológica sobre Espanya», Arxiu d'Etnografia de Catalunya 4-5: 7-226. Tarragona: Publicacions URV. 